\title{
Accelerators in the 21st Century
}

\author{
Frank Zimmermann ${ }^{1, a}$ \\ ${ }^{1}$ CERN, Geneva, Switzerland
}

\begin{abstract}
More than 30,000 accelerators are in operation worldwide. Of these less than $1 \%$ are devoted to basic research. Prominent among the latter are high-energy particle colliders - powerful engines of discovery and precision measurement, which have played an essential role in establishing the standard model of particle physics. Technological innovation has allowed building colliders for ever higher energy and better performance, at decreasing specific cost. New concepts will allow reaching even higher luminosities and energies throughout the coming century. One cost-effective strategy for future collider implementation is staging. For example, a future circular collider could first provide electron-positron collisions, then hadron collisions (proton-proton and heavy-ion), and, finally, the collision of muons. Indeed, cooling-free muon colliders, realizable in a number of ways, promise an attractive and energy-efficient path towards lepton collisions at tens of $\mathrm{TeV}$. While plasma accelerators and dielectric accelerators offer unprecedented gradients, the construction of a high-energy collider based on these advanced technologies still faces a number of challenges. Pushing the accelerating gradients or bending fields ever further, the breakdown of the QED vacuum may, or may not, set an ultimate limit to electromagnetic acceleration.
\end{abstract}

\section{Introduction}

Today more than 30,000 particle accelerators are in operation worldwide. Of these less than $1 \%$ are devoted to basic research. About 5\% of the accelerators are used for applied research, 35\% for medical purposes like the production of radioactive isotopes or cancer therapy, and the remaining $60 \%$ for industrial applications $[1,2]$.

Particle accelerators have proven outstanding engines of discovery: about one third of all physics Nobel prizes since 1939 are linked to particle accelerators [1, 2]. A recent book [3] reviews the past 100 years of accelerator development, accelerator applications, and future accelerator projects for the coming decades.

Particle accelerators are key instruments for many branches of science. Europe is housing 18 synchrotron- and 8 FEL-based light sources, such as ESRF in Grenoble, Diamond at RAL, ELETTRA and FERMI in Trieste, SOLEIL and CLIO in Paris, ALBA-CELLS in Barcelona, PETRA-III, FLASH and the European XFEL in Hamburg, and the SwissFEL at PSI. The most brilliant tunable gamma-ray beam will soon be provided by an accelerator-based laser-Compton source at ELI-NP in Magurele. Across Europe, also several accelerator-based neutron sources are in operation (e.g., ISIS

ae-mail: frank.zimmermann@cern.ch 
at RAL, SINQ at PSI, AMANDE in Cadarache, PTB Braunschweig), and many more under construction oder under consideration (ESS (Lund), FRANZ (JGU Frankfurt), IPHI-NEUTRON (CEA), SONATE (CEA), HBS (Jülich), NOVA ERA (Jülich), LENOS (Legnaro), Bilbao, ... ) [4, 5]. More Nobel prizes from the aforementioned accelerators are "guaranteed". Accelerator-based research has a great impact in all scientific domains.

Concerning medical accelerators, more than 14,000 of these are in use for radiation therapy in hospitals around the world, and more than 500 are operating for isotope production. In addition, there are 19 particle cancer therapy centres in Europe alone.

One important role of industrial accelerators is the analysis and modification of surfaces with many areas of applications (ion implantation, treatment of polymers, sterilisation, environment,...).

The development of accelerators has, however, been driven by basic research, in particular by high-energy physics. The history of high-energy accelerators spans from E.O. Lawrence's first practical cyclotron in 1932, providing 1.22 MeV protons, to today's Large Hadron Colliders (LHC) at CERN, colliding $7 \mathrm{TeV}$ proton beams. During the past century, the accelerators became $3 \times 10^{4}$ larger and they now achieve close to $10^{7}$ times higher energy than 100 years ago. While a large part of this energy rise was accomplished by the increase in size, two to three orders of magnitude were gained by technological improvements.

At the same time, the technology advances have also dramatically reduced the specific construction cost, i.e. the cost per centre-of-mass (c.m.) energy, from about $60 \mathrm{MCHF} /(\mathrm{GeV} \mathrm{c.m}$.) for the CERN Proton Synchotron (PS), first operated in 1959, to $0.3 \mathrm{MCHF} /(\mathrm{GeV}$ c.m.) for the Large Hadron Collider (LHC), in operation since 2008, with both prices expressed in 2008 Swiss francs [CHF] [6, 7] - a cost reduction by a factor 200 over 40 years.

The colliders, which could be realized thanks to this advancing technology, have proven powerful instruments for discovery and precision measurement. All the heavier particles of the standard model were produced at colliders: the tau lepton and charm quark at SPEAR, the top quark at the Tevatron, the gluon at PETRA, the $\mathrm{W}$ and $\mathrm{Z}$ bosons at the $\mathrm{S} p \bar{p} \mathrm{~S}$ collider, and the Higgs boson at the LHC.

The past progress was enabled by the introduction of new concepts (e.g., strong focusing, separate function magnets, colliding beams) as much as by the emergence of new technologies, in particular ones based on superconductivity. PETRA, TRISTAN and LEP-2 started the massive use of superconducting radiofrequency (rf) systems. The Tevatron was the first accelerator based on superconducting magnets. HERA, RHIC, and the LHC used, or use, both superconducting magnets and superconducting rf.

In addition to energy and specific cost, also the accelerator performance was tremendously improved over time: Every year the LHC delivers more luminosity than all the previous hadron colliders together had accumulated over their entire operating history.

\section{Energy Frontier in the 21st Century}

A very large circular hadron collider appears to be the only feasible approach to reach $100 \mathrm{TeV}$ c.m. collision energy in the coming decades. Such collider would offer access to new particles through direct production in the few- $\mathrm{TeV}$ to $30 \mathrm{TeV}$ mass range, far beyond the $\mathrm{LHC}$ reach [8]. It would also provide much-increased rates for phenomena in the sub-TeV mass range and, thereby, a much increased precision compared with the LHC [8].

The centre-of-mass energy reach of a hadron collider is directly proportional to the maximum magnetic field $B$ and to the bending radius $\rho$ :

$$
E_{\text {c.m. }} \propto \rho B .
$$


Therefore, an increase in the size of the collider compared with the LHC by a factor of about 4 and an approximate doubling of the magnetic field yields almost an order of magnitude increase in energy.

Such approach was first suggested in the year 2010 during the High-Energy LHC (HE-LHC) workshop [9]. Now it is the focus of the Future Circular Collider (FCC) study [10], which was launched in response to the 2013 Update of the European Strategy for Particle Physics: 16 Tesla magnets in a 100 $\mathrm{km}$ ring will result in a centre-of-mass energy of $100 \mathrm{TeV}$. This goal defines the overall infrastructure requirements for the FCC accelerator complex. The FCC study scope also includes the design of a high-luminosity $\mathrm{e}^{+} \mathrm{e}^{-}$collider (FCC-ee), as a possible first step — with a remarkably rich physics programme [11] —, as well as a proton-electron collision option (FCC-he) at one interaction point (IP), where a $60 \mathrm{GeV}$ electron beam from an energy recovery linac is collided with one of the two 50 $\mathrm{TeV}$ proton beams circulating in the FCC-hh. The design of a higher-energy hadron collider in the LHC tunnel based on FCC-hh magnet technology — the so-called High-Energy LHC (HE-LHC) is yet another part of the FCC study.

Figure 1 compares the time lines of various past and present circular colliders at CERN with a projected time line for the FCC, indicating a need for fast progress.
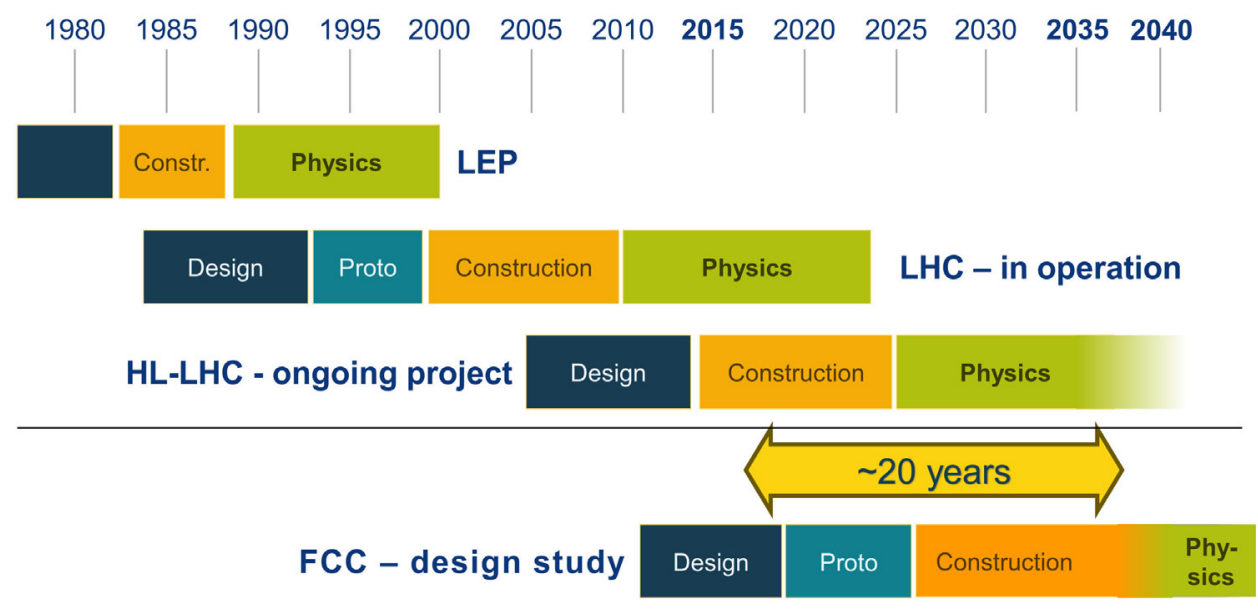

Figure 1. Time lines of several past, present and future circular colliders at CERN, distinguishing periods of design, prototyping, construction, and physics exploitation.

CEPC and SppC are two colliders similar to FCC-ee/FCC-hh, which are being studied by a collaboration centred at IHEP Beijing [12]. These two machines have a similar circumference of about 100 $\mathrm{km}$. Several possible locations in China are under study. The $\mathrm{e}^{+} \mathrm{e}^{-}$collider CEPC is designed with a maximum centre-of-mass energy of $240 \mathrm{GeV}$, and a 3 or 200 times lower luminosity than FCC-ee, at the Higgs production peak and on the $\mathrm{Z}$ resonance, respectively. The $\mathrm{SppC}$ hadron collider relies on $12 \mathrm{~T}$ (later $24 \mathrm{~T}$ ) iron-based high-temperature superconducting magnets, which could be installed in the same tunnel as the CEPC.

Table 1 shows key parameters of FCC-hh, SppC, and HE-LHC, together with the design values of the present LHC and its luminosity upgrade (HL-LHC). Table 2 compares parameters for FCC-ee and CEPC at different operating energies with those of LEP-2 and SuperKEKB.

Construction cost of future projects can, and should, be minimized by [13, 14]: (1) reducing the cost of the components, e.g., superconductor and high-field magnet for the hadron colliders; (2) 


\begin{tabular}{|c|c|c|c|c|c|c|}
\hline parameter & \multicolumn{2}{|c|}{ FCC-hh } & \multicolumn{2}{|c|}{ SppC } & HE-LHC & (HL-)LHC \\
\hline c.m. energy $[\mathrm{TeV}]$ & \multirow{2}{*}{\multicolumn{2}{|c|}{$\begin{array}{c}100 \\
16\end{array}$}} & 75 & 150 & 27 & 14 \\
\hline dipole field [T] & & & 12 & 24 & 16 & 8.3 \\
\hline circumference $[\mathrm{km}]$ & \multicolumn{2}{|c|}{97.8} & \multicolumn{2}{|c|}{100} & 26.7 & 26.7 \\
\hline beam current $[\mathrm{A}]$ & \multicolumn{2}{|c|}{0.5} & 0.77 & - & 1.12 & (1.12) 0.58 \\
\hline particles/bunch [10 $\left.10^{11}\right]$ & \multicolumn{2}{|c|}{1} & 1.5 & - & 2.2 & (2.2) 1.15 \\
\hline bunch spacing [ns] & \multicolumn{2}{|c|}{25} & 25 & - & 25 & 25 \\
\hline normalized emittance $[\mu \mathrm{m}]$ & \multicolumn{2}{|c|}{$2.2(1.1)$} & 3.16 & - & $2.5(1.25)$ & (2.5) 3.75 \\
\hline IP beta function $[\mathrm{m}]$ & 1.1 & 0.3 & 0.71 & - & 0.25 & $(0.15) 0.55$ \\
\hline luminosity $\left[10^{34} \mathrm{~cm}^{-2} \mathrm{~s}^{-1}\right]$ & 5 & 30 & 10 & 100 & 25 & (5) 1 \\
\hline events per crossing & 170 & 1000 & $\sim 300$ & - & 800 & (135) 27 \\
\hline SR power/beam [kW] & \multirow{2}{*}{\multicolumn{2}{|c|}{$\begin{array}{c}2400 \\
1.1\end{array}$}} & 1130 & - & 100 & (7.3) 3.6 \\
\hline longitudinal damp. time $[\mathrm{h}]$ & & & 2.4 & - & 3.6 & 25.8 \\
\hline initial burn-off time [h] & 17 & 3.4 & 13 & - & 3.0 & (15) 40 \\
\hline
\end{tabular}

Table 1. Parameters of future hadron colliders, the LHC and its HL-LHC upgrade

\begin{tabular}{|l|c|c|c|c|c|c|c|}
\hline parameter & \multicolumn{3}{|c|}{ FCC-ee } & \multicolumn{2}{c|}{ CEPC } & LEP-2 & SKEKB \\
\hline beam energy [GeV] & 45.6 & 120 & 182.5 & 45.5 & 120 & 104 & $4 / 7$ \\
\hline circumference [km] & \multicolumn{3}{|c|}{97.8} & \multicolumn{2}{|c|}{100} & 26.7 & 3.0 \\
\hline beam current [mA] & 1390 & 29 & 5.4 & 19 & 74 & 3 & $\sim 3000$ \\
particles/bunch [10 $\left.{ }^{11}\right]$ & 1.7 & 1.5 & 2.8 & 0.1 & 1.0 & 4.2 & $\sim 1$ \\
horizontal emittance [nm] & 0.3 & 0.6 & 2 & 0.2 & 1.3 & 22 & 4 \\
vertical emittance [pm] & 1 & 1 & 3 & 0.9 & 4 & 250 & 10 \\
horizontal IP beta [m] & 0.15 & 0.3 & 1 & 0.17 & 0.17 & 1.2 & 0.03 \\
vertical IP beta [mm] & 0.8 & 1 & 2 & 2 & 2 & 50 & 0.3 \\
luminosity [10 $\mathrm{cm}^{34} \mathrm{~s}^{-1}$ ] & $>200$ & $>7$ & $>1.3$ & 1.1 & 2.0 & 0.01 & 80 \\
\hline
\end{tabular}

Table 2. Parameters of future $\mathrm{e}^{+} \mathrm{e}^{-}$colliders, LEP-2 and SuperKEKB.

building on a site with an existing infrastructure and injector complex, e.g., on the CERN site; and (3) staging, e.g., FCC-ee followed by FCC-hh, and possibly by a muon collider FCC- $\mu \mu$ (see below).

\section{Synchrotron Radiation}

Synchrotron radiation (SR) is an obstacle on the path to higher energy, as the resulting energy loss per turn increases with the fourth power of a charged particle's energy. For FCC-ee, synchrotron radiation limits the maximum attainable c.m. energy to about $400 \mathrm{GeV}$ since at higher energy the required rf voltage could no longer be provided by conventional rf technology. In addition, by design, the FCC-ee beams emit a total of 100 MW SR power at all energies, which must be sustained by efficient rf power sources fed from the grid.

However, synchrotron radiation does not only afflict the electron and positron machines. The synchrotron radiation of the FCC-hh hadron collider amounts to about $5 \mathrm{MW}$, emitted inside the cryogenic arcs. Indeed, the power consumption of the FCC-hh hadron collider is likely to be dominated 
by the effects of this synchrotron radiation. Namely, the heat extraction from the beam screen inside the cold magnets and from the cold bore itself requires well above $100 \mathrm{MW}$ of electric power for the cryogenics plants. In addition, of order $10 \mathrm{MW}$ electric power is required for the rf system to maintain the energy of the stored hadron beams.

Several possible approaches exist for eliminating, avoiding, or at least reducing the synchrotron radiation, which we discuss in the following subsections.

\subsection{Beam Shaping or Beam Pipe Tayloring}

The synchrotron radiation (SR) in circular $\mathrm{e}^{+} \mathrm{e}^{-}$and hadron colliders could, in principle, be suppressed by either of the following two approaches.

1. Shaping the beam: Classically a time-invariant beam — like a constant-current loop — does not radiate, i.e., it does not emit any electromagnetic waves. For the same reason a quieter beam or a crystalline beam would exhibit less synchrotron radiation. In particular, homogenization of the beam on the scale of the radiation wavelength results in destructive interference and consequently subradiance, i.e., lower intensity than expected from spontaneous radiation [1517].

Suppression of shot noise and the resulting strong reduction in undulator radiation were observed experimentally at the SLAC NLCTA [17], where a "dispersive method" [18] was used to suppress the beam's shot noise. Namely, the electron beam's Coulomb forces first generated an energy modulation while the beam propagated through a so-called interaction region (23 $\mathrm{m}$ long). Next, passing through a donwstream magnetic chicane yielded a "quieter" electron beam, which emitted subradiantly in an undulator. For the optimum chicane setting, a suppression factor of 2.6 was observed in the integrated far-field radiation from the undulator. The SLAC NLCTA experiment "demonstrated the feasibility of noise suppression as a practical tool in accelerator physics" [17].

For the extreme case of a highly ordered one-dimensional crystalline beam, attainable by beam cooling, the suppression can be much higher. Distributing $10^{6}$ ultrarelativistic charged particles uniformly around a storage ring leads to a factor 50 reduction in the total SR power [19].

2. Tayloring the beam-pipe boundary: A large bending radius $\rho$ combined with a small chamber suppresses SR emission at long wavelengths [20,21].

Specifically, radiation is shielded at wavelengths $\lambda \geq 2 \sqrt{d^{3} / \rho}$, where $d$ denotes the beam-pipe diameter [22]. For example, with $d=10 \mathrm{~mm}$ and $\rho=1 \mathrm{~km}$ shielding occurs for $\lambda>600 \mathrm{~nm}$ (or photon energies below $2 \mathrm{eV}$ ), while with $d=0.1 \mathrm{~mm}$ and $\rho=10 \mathrm{~km}$ the radiation is suppressed for $\lambda>2 \mathrm{pm}$ (or photons energies up to $600 \mathrm{keV}$ ).

One experimental evidence for suppression of incoherent synchrotron radiation comes from RHIC, where fully stripped gold ion $\left(\mathrm{Au}^{+79}\right)$ experienced a nearly total suppression of synchrotron radiation (energy loss per turn reduced by more than a factor ten) at an energy of 70 $\mathrm{GeV} /$ nucleon, and still a reduction by a factor larger than two at $100 \mathrm{GeV} /$ nucleon [23]. The RHIC measurements of radiation suppression by vacuum-chamber shielding are consistent with the theoretical expectation [23].

The shielding effect can be enhanced by further decreasing the dimensions of the vacuum chamber. Beam-pipe coating with a high-temperature superconductor [24-26] (to ensure an acceptable impedance in spite of small beam-pipe dimensions) might allow for millimeter-, micro-, or even nano-chambers. The use of nanotubes or crystal structures would enable even smaller 
dimensions and still better shielding. A hollow plasma channel would be yet another candidate low-aperture "chamber" shield.

\subsection{Linear Colliders}

The avoidance or reduction of synchrotron radiation has been the key motivation for proposing a transition from circular to linear $\mathrm{e}^{+} \mathrm{e}^{-}$colliders. Linear colliders contain no arcs for bending, or at least much less bending than a circular ring, and the beam only passes a single time, minimizing the effects of synchrotron radiation. Presently proposed projects include the International Linear Collider (ILC) [27] and the Compact Linear Collider (CLIC) [28].

Figure 3 shows the projected total luminosity of four proposed future high-energy $\mathrm{e}^{+} \mathrm{e}^{-}$colliders as a function of c.m. energy. The circular collider FCC-ee promises highest luminosity up to a c.m. energy of about $400 \mathrm{GeV}$. The lower target luminosities for CEPC, though essentially the same machine as FCC-ee, are matched to its less ambitious physics goals. It is not planned to operate CEPC at the top quark threshold. At higher energies, above $400 \mathrm{GeV}$, linear colliders reveal their potential. ILC and CLIC can cover the c.m. energy range up to 1 and $3 \mathrm{TeV}$, respectively,

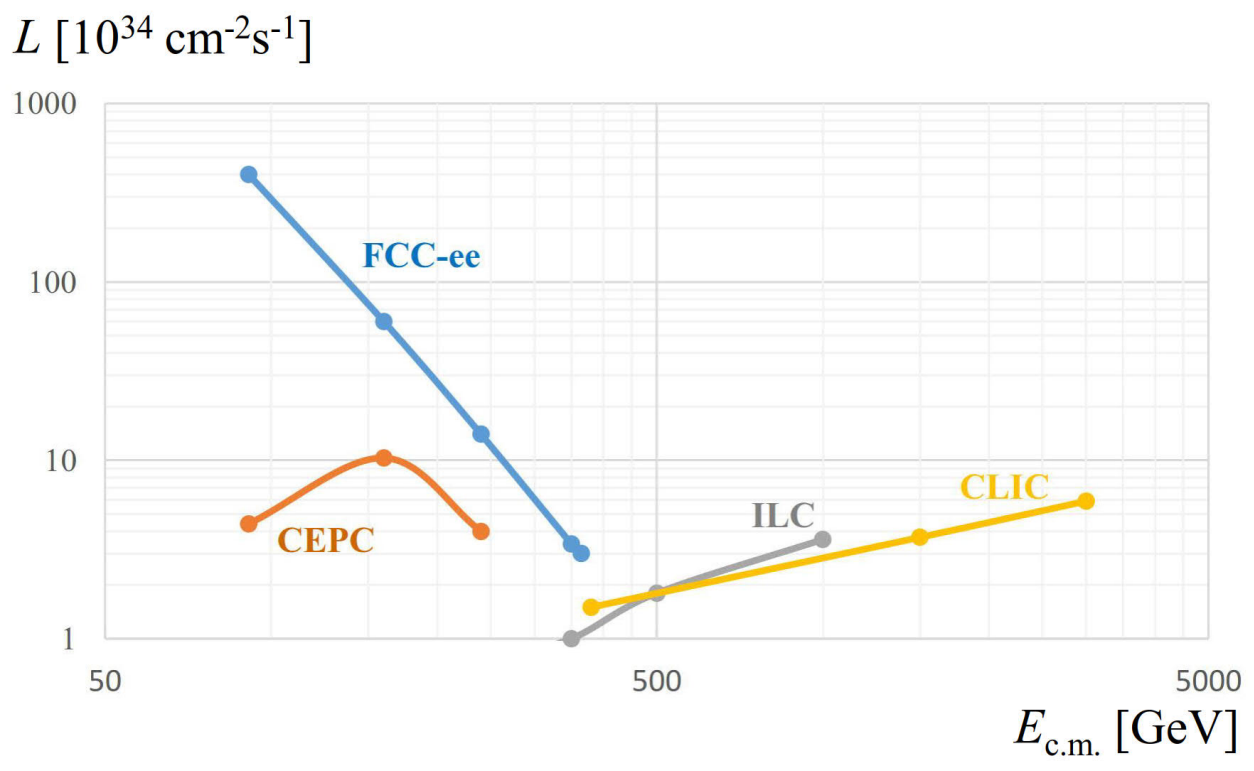

Figure 2. Total luminosity of various proposed highest-energy $\mathrm{e}^{+} \mathrm{e}^{-}$colliders as a function of c.m. energy. For the circular machines, FCC-ee and CEPC, two interaction points are considered, while the linear colliders, ILC and CLIC, can only serve a single interaction point each.

Linear colliders apear intrinsically inefficient, as the entire beam is disposed, and its energy lost, after only a single collision. In addition, synchrotron radiation is still present during the collision (here called "beamstrahlung"), where it becomes a significant limitation at multi-TeV beam energies.

Future advanced variants of linear colliders with higher gradient, and, therefore, of smaller size, are proposed to be based on laser- or beam-driven plasma wake-field acceleration schemes (LPA [29] or PWFA [30-32]) or on dielectric laser acceleration (DLA [33]) schemes. At present such acceleration schemes, which promise 10-1000 times higher accelerating gradients than conventional rf 
structures, still tend to have a non-negligible specific cost, currently as high as the super- or normalconducting rf systems [13], if not higher. Their luminosity per wall-plug power might also increase with beam energy less strongly than required by particle physics [34]. Preserving the quality of a positron beam during plasma acceleration may prove yet another challenge [35].

\subsection{Muon Colliders}

Another forward-looking approach consists in changing the particle type from $\mathrm{e}^{+} \mathrm{e}^{-}$to muons. Indeed such muon-collider schemes [34] promise a high efficiency, as we will discuss in the following.

\section{Energy Efficiency}

Figure 3 demonstrates that up to the top quark threshold $(\sim 380 \mathrm{GeV})$ the circular collider FCC-ee offers by far the highest luminosity at the lowest electric input power, revealing the FCC-ee characteristics as a truly "green" accelerator. The FCC-ee would naturally be followed by FCC-hh, which spans a wide range of parton collision energies at high luminosity, for a total electric input power similar to the one of FCC-ee. A possible third stage would be a muon collider, the only lepton-collider approach which offers a high luminosity at higher energy [34], as is required for particle-physics explorations.

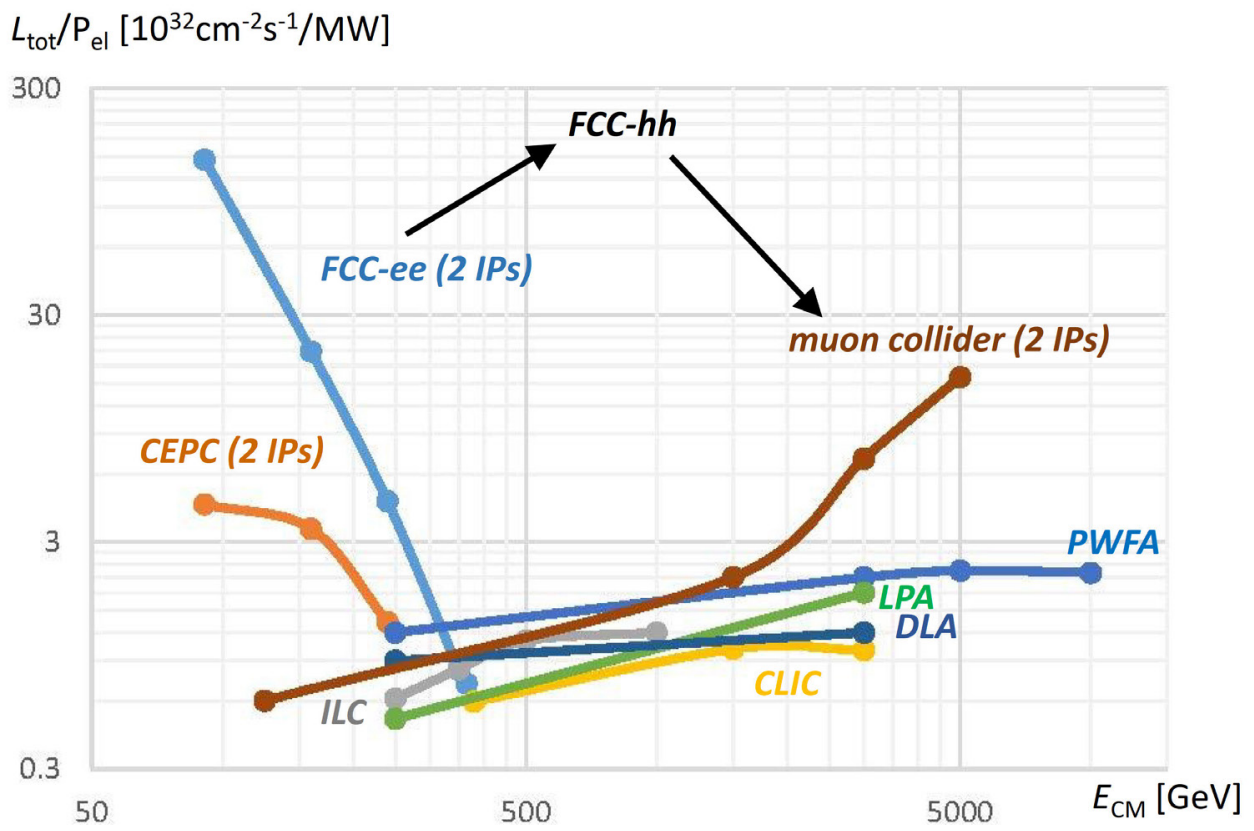

Figure 3. Lepton-collider luminosity per electrical power for different accelerator projects and technologies, hinting that the staged approach FCC-ee $(\rightarrow$ FCC-hh $) \rightarrow$ FCC- $\mu \mu$ would deliver highest possible luminosity at c.m. energies from $91 \mathrm{GeV}$ to $>5 \mathrm{TeV}$ in the most efficient way $[34,36]$.

Figure 4 sketches one example configuration for converting the LHC-FCC complex into a muon collider, using the concept of muon production in laser-Compton collisions with a partially stripped heavy-ion (PSI) beam, the so-called Gamma Factory [37]. 


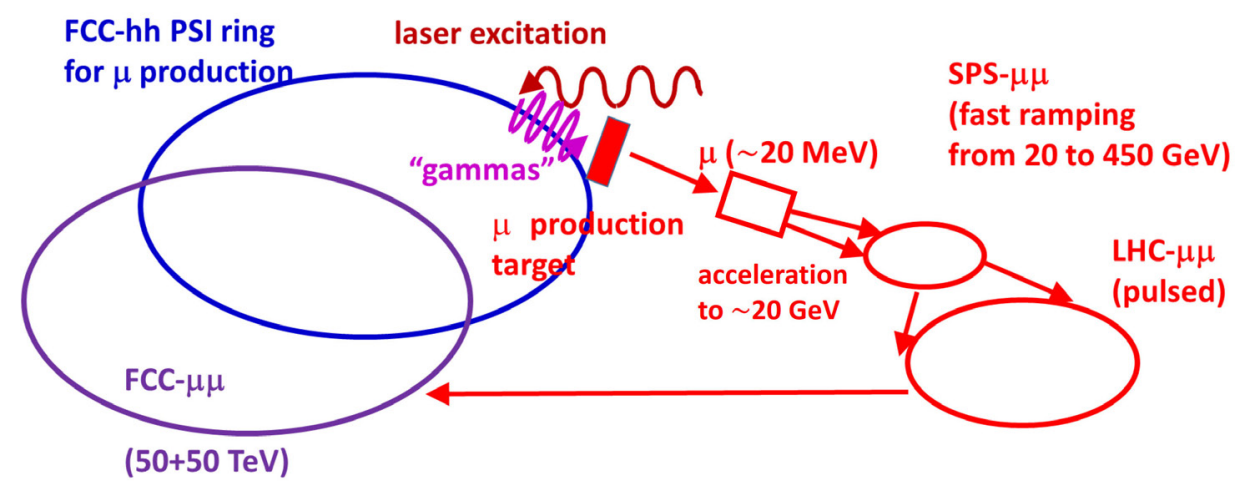

Figure 4. Schematic of a $100 \mathrm{TeV}$ muon collider, FCC- $\mu \mu$ based on the LHC/FCC complex and the Gamma Factory concept, where laser-pulse collision with a partially stripped ion beam stored in one of the FCC-hh rings is used for $\mu^{ \pm}$production.

An alternative approach would rely on low-emittance muon beam generation by positron annihilation in a $\sim 45-\mathrm{GeV}$ storage ring [38] (which could be FCC-ee or its top-up booster, already optimized for $\mathrm{Z}$ pole operation at the same energy), where the positron beam would be produced either from a conventional source or, in higher intensity, also by the Gamma-Factory approach.

Whatever the scheme chosen, the muon beams would be collided in one of the two LHC or FCC rings, while the second ring (or the other one of the two accelerators, respectively) might potentially be used to store partially stripped heavy-ion beams for muon or positron production. Remaining issues, still to be tackled, include the muon stacking, rapid acceleration, muon synchrotron radiation and neutrino radiation.

\section{Outlook}

An ultimate limit on electromagnetic acceleration may be set by the Sauter-Schwinger critical field, above which the QED vacuum breaks down. This critical field is equal to $E_{\mathrm{cr}} \approx 10^{12} \mathrm{MV} / \mathrm{m}$ or $B_{\mathrm{ct}} \approx 4.4 \times 10^{9} \mathrm{~T}$. Assuming these fields, the Planck scale of $10^{28} \mathrm{eV}$ can be reached by a circular or linear collider with a size of about $10^{10} \mathrm{~m}$, or about a tenth of the distance between earth and sun, for either type of collider (!). A solar-system Planck-energy linear collider was considered previously, and it was judged to be "not an inconceivable task for an advanced technological society" [39].

The future holds other tantalizing accelerator challenges, such as (1) the possible use of accelerators for the detection or generation of gravitational waves [42-44]; (2) the possibility of constructing a high-field crystal [45, 46] or nano-tube [47] accelerator (for acceleration [45] or bending [46, 48] — see Fig. 5); (3) the strategy for approaching (or exceeding) the Planck scale [41], and the possible acceleration beyond the Schwinger limit e.g., by developing a "quantum plasma accelerator" [40], or using techniques of entanglement [49].

\section{Acknowledgements}

I would like to thank the organizers of ICNFP2017, especially Dr. Mike Koratzinos, for inviting me to the conference and for continuous encouragement. This work was supported, in part, by the European Commission under the HORIZON2020 Integrating Activity project ARIES, grant agreement 730871. 


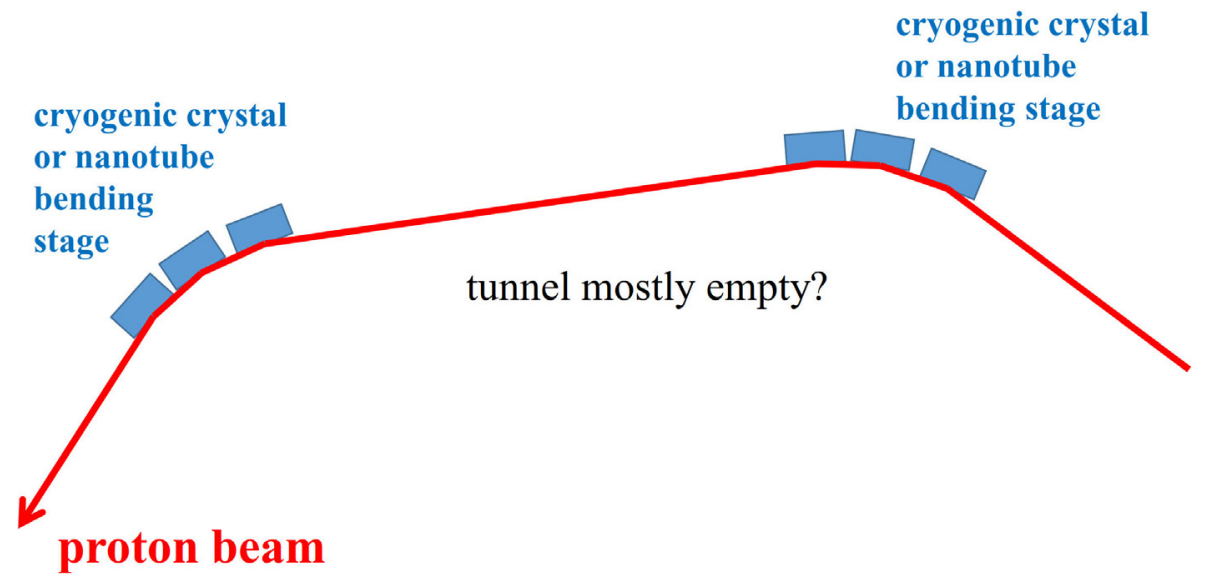

Figure 5. Schematic of a circular crystal or nanotube collider [48].

\section{References}

[1] M. Vretenar, Accelerator Science in the XXIst century and the role of ARIES, ARIES Kick-Off meeting, Geneva, 4-5 May 2017 (2017)

[2] A. Faus-Golfe, M. Vretenar et al., Applications of Particle Accelerators in Europe, EuCARD-2 APAE document, available at http://apae.ific.uv.es/apae/

[3] O. Brüning and S. Myers (Eds.), Challenges and Goals for Accelerators in the XXI Century (World Scientific, Singapore, 2016)

[4] J. Suverink, private communication (2017)

[5] I.S. Anderson et al., Physics Reports 654, 1-58 (2016)

[6] P. Lebrun, Costing High-Energy Accelerator Systems, 4th EuCARD AccNet RFTech Workshop, 25-26 March 2013, Annecy (2013)

[7] F. Zimmermann, Strategy for Future Proton and Electron Facilities in Europe, EuCARD Report EuCARD-REP-2013-008 (2013), http: //cds . cern.ch/record/1708761

[8] M. Mangano (Ed.), Physics at the FCC-hh, a 100 TeV pp Collider, CERN Yellow Reports: Monograph CERN-2017-003-M (2017), also available as arXiv:1710.06353

[9] E. Todesco and F. Zimmermann (Eds.), Proceedings, EuCARD-AccNet-EuroLumi Workshop: The High-Energy Large Hadron Collider (HE-LHC10): Villa Bighi, Malta, Republic of Malta, October 14-16, 2010, CERN Yellow Report CERN-2011-003 (2011), also available as arXiv:1111.7188

[10] M. Benedikt and F. Zimmermann, Towards Future Circular Colliders, Journal of the Korean Physical Society 69, 893 (2016)

[11] The TLEP Design Study Working Group (M. Bicer et al.), First Look at the Physics Case of TLEP, J. High Energ. Phys. 01, 164 (2014), also available as arXiv:1308.6176

[12] J. Gao, Int. J. Mod. Phys. A 32, no. 2, 1746003 (2017)

[13] V. Shiltsev, JINST 9, T07002 (2014)

[14] F. Zimmermann (Ed.), Strategy for Future Extreme Beam Facilities, EuCARD Monograph no. XLIV (Institute of Electronic Systems, Warsaw University of Technology, Warsaw, 2017) 
[15] R.H. Dicke, Phys. Rev. 93, 99 (1954)

[16] A. Gover and E. Dyunin, Phys. Rev. Lett. 102, 154801 (2009)

[17] D. Ratner et al., Phys. Rev. ST Accel. Beams 18, 050703 (2015)

[18] D. Ratner, Z. Huang, and G. Stupakov, Phys. Rev. ST Accel. Beams 14, 060710 (2011)

[19] H. Primack and R. Blümel, Phys. Rev. E 60, 957 (1999)

[20] J. Schwinger, On Radiation by Electrons in a Betatron, LBNL Report LBNL-39088 (1945)

[21] J.S. Nodvick and D.S. Saxon, Phvs. Rev. 96, 180 (1954)

[22] R.L. Warnock, Shielded Coherent Synchrotron Radiation and its Effect on Very Short Bunches, SLAC Report SLAC-PUB-5375 (1990)

[23] N.P. Abreu et al., Energy Loss of Coasting Gold Ions and Deuterons in RHIC, Proc. EPAC'08, Genoa, Italy (2008)

[24] O. Brüning, B. Goddard, M. Mangano, S. Myers, L. Rossi, E. Todesco, F. Zimmermann, High Energy LHC Document prepared for the European HEP Strategy Update, CERN Report CERNATS-2012-237 (2012).

[25] W. Barletta, M. Battaglia, M. Klute, M. Mangano, S. Prestemon, L. Rossi, P. Skands L. Rossi, Nucl. Instr. Methods Phys. Research A 764, 352 (2014)

[26] S. Calatroni, IEEE Trans. Appl. Supercond. 26, 3500204 (2016)

[27] T. Behnke et al., The International Linear Collider Technical Design Report, ILC Report ILCREPORT-2013-040 (2013)

[28] P. Lebrun et al., The CLIC Programme: Towards a Staged e+e- Linear Collider Exploring the

Terascale: CLIC Conceptual Design Report, CERN Yellow Report CERN-2012-005 (2012)

[29] E. Esarey, C.B. Schroeder, and W.P. Leemans, Rev. Mod. Phys. 81, 3, 1229-1285 (2009)

[30] C. Joshi, AIP Conference Proceedings 737, 3 (2004)

[31] A. Martinez de la Ossa et al., Phys. Rev. Accel. Beams 20, 9, 091301 (2017)

[32] Y. Li et al., Phys. Rev. Accel. Beams 20, 101301 (2017)

[33] R. England et al., Rev. Mod. Phys. 86, 4, 1337-1389 (2014)

[34] J.P. Delahaye et al., A Staged Muon Accelerator Facility for Neutrino and Collider Physics, Fermilab Report FERMILAB-CONF-14-211-APC-E-T (2015)

[35] K.V. Lotov, Physics of Plasmas, 14, 023101 (2007)

[36] A. Blondel and P. Janot, private communication (2017)

[37] M.W. Krasny, The Gamma Factory Proposal for CERN, arxiv:1511.07794 (2015)

[38] M. Boscolo, M. Antonelli, R. Di Nardo and P. Raimondi Nucl. Instr. Meth. A 807, 101-107 (2015)

[39] P. Chen and R.J. Noble, AIP Conf. Proc. 398, 273-285 (1997)

[40] F. Zimmermann, unpublished (2017)

[41] S.B. Giddings, Beyond the Planck Scale, CERN Report CERN-PH-TH/2009-193, also arXiv:0910.3140, (2009)

[42] V.A. Braginsky, C.M. Caves, and K.S. Thorne, Phys. Rev. D 15, 8, 2047 (1977)

[43] R. D.'Agnolo, Storage Rings as Antennas for Gravitational Waves?, EuCARD-2 XBEAM Strategy Workshop, Valencia, 13-17 February 2017 (2017)

[44] M. Zanetti and F. Zimmermann, ARIES Work Package 6 Accelerator Performance and Novel Concepts, Task 6.6 (2017)

[45] V. Shiltsev, Phys. Usp. 55, 965-976 (2012)

[46] W. Scandale et al., Phys. Rev. Lett. 101, 16, 164801 (2008) 
[47] Y.-M. Shin, Plasmon-Driven Acceleration in a Photo-Excited Nanotube, arXiv 1709:02419 (2017)

[48] F. Zimmermann, Nucl. Instrum. Methods Phys. Res. B 355, 4-10 (2015)

[49] S. Brooks, View on Future Accelerators/Concepts, EuCARD-2 EuroNNAc-XBEAM joint workshop F3iA 2016 Focus: Future Frontiers in Accelerators, 5-9 December 2016, Scharbeutz, Germany (2016), http: //indico.desy.de/indico/event/15657 